\title{
Bundestagswahl: Die Zweifel fressen sich durch
}

Die deutsche Politik hat mit der Bundestagswahl Entwicklungen nachvollzogen, die in anderen europäischen Ländern schon länger im Gang sind: Schrumpfung der ehemaligen „Volksparteien“ der rechten und linken Mitte, ein mögliches Abrutschen insbesondere der alten Arbeiterpartei in die Bedeutungslosigkeit, Fragmentierung des Parteiensystems, Aufstieg einer neo-nationalistischen Rechtspartei mit Hilfe reaktivierter Nichtwähler (35\% der AfD-Stimmen). Merkels Projekt einer Verlängerung der "Willkommenskultur" als Grundlage einer liberal-bürgerlichen Koalition mit den Grünen ist gescheitert, ebenso der Versuch der SPD, auf dem Trittbrett der „Weltoffenheit" in eine post-proletarische Zukunft zu fahren. Gemeinsame Verluste: 13,7\%.

Diesmal kann es lange dauern, bis die neue Regierung steht, und danach wird es bald um das Ende der Kanzlerschaft von Angela Merkel gehen. Regiert werden wird bis dahin eher wenig. Für die Grünen ist Jamaika die letzte Chance; sie werden deshalb schlucken, was innen eingeschenkt wird. Der FDP wird der Bundesverband der Deutschen Industrie (BDI) notfalls gut zureden. Das Problem ist die CSU; sie muss 2018 ihre absolute Mehrheit in Bayern verteidigen und dazu ihre Wählerschaft um ein Viertel aufstocken. Holen kann sie die Stimmen nur bei der AfD. Substanzielle europapolitische Konzessionen wird sie deshalb so wenig mittragen können wie offene Obergrenzen jeder Art, selbst wenn sie wie jetzt nur auf dem Papier stehen. Beide Male kann sie auf die FDP als Verbündeten und auf die Grünen, mit Merkel im Hintergrund, als Widersacher zählen. Bei anderen Themen (innere Sicherheit!) wird sie es mit beiden zugleich aufnehmen müssen. Unmittelbar nach der bayrischen Landtagswahl wird dann die Frage zu beantworten sein, an wen Merkel in der Mitte der Legislaturperiode den Stab zu übergeben hat und ob sie bei der Auswahl des oder der Glücklichen noch mitreden darf. Große Sprünge wird sie weder vorher noch nachher machen können.

Was kann die SPD tun, um dem Schicksal ihrer Schwesterparteien in Frankreich und den Niederlanden zu entgehen? Einen Corbyn hat sie nicht. Im Osten ist sie eine Splitterpartei (Rang 4 hinter CDU, AfD und Linke), im Süden schon länger eine Sekte. Im Wahlkampf hat sich Schulz unermüdlich bei Merkel als Vizekanzler beworben; vielleicht kommt sie darauf zurück und die SPD besinnt sich „aus staatspolitischer Verantwortung". Solange man das für möglich halten kann, stärkt das Merkels Position gegenüber FDP und CSU. „Kein Abschied auf der Welt", so schon Talleyrand, „fällt schwerer als der Abschied von der Macht". Nach der Wahl beklagte sich Schulz, dass die Bundeskanzlerin Diskussionen verweigert habe und überhaupt ein „Ideenstaubsauger" sei; bei der SPD kann sie freilich nichts abgesaugt haben, und mit irgendwie kontroversen Diskussionsbeiträgen sind die Steinmeiers, Steinbrücks, Gabriels und Oppermanns nicht auffällig geworden. Im Gegenteil hat sich die SPD in den letzten Jahren unermüdlich daran beteiligt, aufkommende Fragen von Bedeutung und Interesse (Regelung der Einwanderung, Zukunft der Nationalstaaten in Europa, Um- und Weiterbau des Euro) als AfD-Fragen zu brandmarken und damit nicht nur sich selbst zu entwaffnen, sondern interessierte Wähler direkt an die AfD zu verweisen. Wie danach die von der SPD angekündigte „entschiedene Opposition“ aussehen kann, muss man sehen. Erste Äußerungen lassen erwarten, dass man, in der Fußballersprache, als fallende Sechs vor der Merkel'schen Viererkette antifaschistische Vorhut gegen die AfD zu spielen beabsichtigt. Allerdings geriete man dabei in Wettbewerb mit der Linken, die es ebenfalls an Bekundungen von Scham und Abscheu nicht fehlen lassen wird. Alternativ könnte man die gemeinsame Zeit in der Opposition, mit

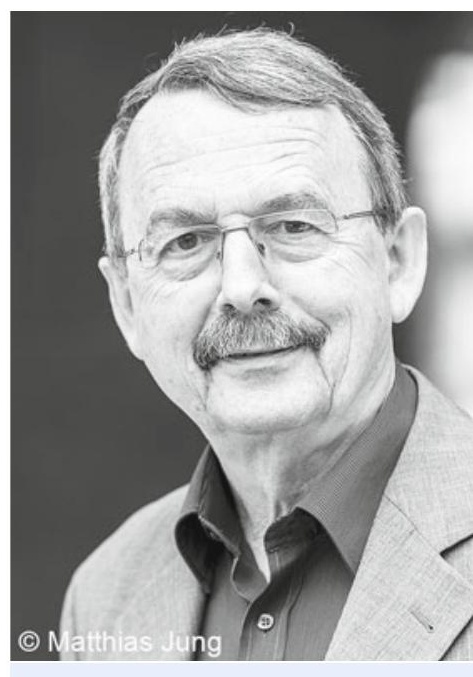

Prof. em. Dr. Dr. h.c. Wolfgang Streeck ist Direktor emeritus am Max-Planck-Institut für Gesellschaftsforschung (MPIfG) in Köln. 
immerhin zusammen rund 30 Stimmenprozenten, zur gemeinsamen Bekämpfung der Regierung und langfristigen Entwicklung einer linken Politikalternative nutzen. Wer sich davor fürchtet, mag beruhigt sein: wahrscheinlich ist das nicht.

Zur Krise der SPD gehört, dass sie auch ein Vierteljahrhundert nach der Wiedervereinigung im Osten nicht hat Fuß fassen können. Dort haben, bei niedrigerer Wahlbeteiligung, die beiden Outcasts des deutschen Parteiensystems, die Linke und die AfD, zusammen 38,0\% der Stimmen, die AfD erstmals mehr als die mittlerweile wohl zu „verwestlichte“ Linke, bei hoher gegenseitiger Austauschbarkeit der Wähler. Zweifellos besteht hier ein, komplex vermittelter, Zusammenhang mit dem Umstand, dass das Pro-Kopf-Einkommen im Osten seit langem bei etwa drei Vierteln des nationalen Durchschnitts stagniert, was mannigfache kulturelle Konflikte befeuert. Beunruhigen sollte, dass seit Jahrzehnten hohe Fördermittel in den Osten fließen, ohne dass die Lücke sich schlösse. Man fragt sich, warum die Wirtschaftswissenschaften keine politisch praktikablen Rezepte für eine regionale Ungleichheiten ausgleichende Regionalpolitik anzubieten haben und selbst unter den vergleichsweise idealen deutschen Bedingungen nicht zu verhindern sein soll, dass ein Teil des Landes wirtschaftlich und politisch zu einer Art Mezzogiorno wird. Denkt man hier weiter, so kann man auf die Frage kommen, ob zwischen der ostdeutschen Malaise und der deutschen Währungsunion von 1990 ein Zusammenhang bestehen könnte: ob eine weiche Ökonomie durch Einführung einer harten Währung, noch dazu bei einem „falschen“ Wechselkurs, so geschädigt werden kann, dass sie trotz hoher Transfers zu jahrzehntelanger Rückständigkeit verurteilt ist. Wenn dies so wäre, würde es nichts Gutes für die Europäische Währungsunion und die deutsche Politik in ihr bedeuten. Kann man wirklich hoffen, dass internationale Umverteilung in Europa mehr bewirken kann als nationale Umverteilung in Deutschland - und dass in den europäischen Südländern andere, weniger systemkritische politische Reaktionen eintreten werden als in den deutschen Ostländern?

Die Regierung Merkel IV wird mit umfangreichen, politisch nachdrücklich vorgetragenen französischen Forderungen nach einem Ausbau der Eurozone zu einem wirtschaftlichen Ausgleichsapparat für den Mittelmeerraum einschließlich Frankreichs konfrontiert sein, finanziert im Wesentlichen durch Deutschland. Dies wird, wenn es der alten SPD-Führung nicht doch noch gelingt, die Große Koalition zu erneuern, an der CSU, aber wahrscheinlich schon an der FDP scheitern. (Berichten zufolge soll Macron geäuBert haben, wenn die FDP der nächsten deutschen Regierung angehöre, sei er „tot“.) Aber selbst wenn Merkel einen Weg fände, die französischen Forderungen zu erfüllen, lassen die Erfahrungen mit der deutsch-deutschen Währungsunion erwarten, dass auch ein an die Grenzen des politisch Möglichen gehender Mitteleinsatz nicht mehr bewirken kann, als ein weiteres Anwachsen der Einkommenslücke zu verhindern.

Dies mag bei der kurzen Perspektive und überwiegenden Symbolhaftigkeit heutiger Politik vorerst nicht zählen. Andererseits hat gerade die diesjährige Bundestagswahl gezeigt, welche langfristigen politischen Folgen unrealistische Versprechungen („,blühende Landschaften") haben können. Wenn man erlebt hat, wie eine Politik, die durch Verbreitung von Illusionen Zeit zu kaufen sucht, riskiert, in dauerhaftem Unfrieden zu enden, dann muss man begrüßen, dass die latenten Zweifel an der symbol- und situationsgetriebenen Merkel-Steinmeier-Gabriel-Schulz-Europapolitik sich im Wahlprozess durchgefressen haben (im Desaster von CDU und SPD, der Stagnation der Grünen, dem Aufstieg der FDP und dem Einrücken der CSU in eine Vetoposition).

Wolfgang Streeck

Max-Planck-Institut für

Gesellschaftsforschung ws@mpifg.de
Eine zeitweilige europapolitische Lähmung der neuen Bundesregierung könnte der Beginn einer dringend nötigen Reflexionsphase im Prozess der europäischen Integration sein: schlechte Zeiten für Macron, aber vielleicht gute für Europa und, damit, sogar Frankreich. 